\title{
Design, synthesis and evaluation of novel quinolyl chalcones as antibacterial agents
}

\author{
Kishor H. Chikhalia, ${ }^{a}$ Mayank J. Patel,, ${ }^{*}$ and Dhaval B. Vashi ${ }^{\text {b }}$ \\ ${ }^{a}$ Department of Chemistry, School of Science, Gujarat University Navrangpura, \\ Ahemadabad-380 009, Gujarat State, India \\ ${ }^{b}$ Department of Chemistry, Faculty of Science Synthetic Organic Chemistry Research \\ Laboratory, Veer Narmad South Gujarat University, Udhna-magdalla Road, \\ Surat (west)-395 007, Gujarat State, India \\ E-mail:ptl.mayank@gmail.com
}

\begin{abstract}
Some new substituted quinolinyl chalcones were synthesized and evaluated for their in vitro antimicrobial activity against Gram positive and Gram negative strains using a microdilution procedure. Synthesized compounds $10 \mathbf{a}-\mathbf{g}$ and $\mathbf{1 3 h}-\mathbf{q}$ prove to be effective with $\mathrm{MIC}\left(\mathrm{mg} \mathrm{ml}^{-1}\right)$, among them 10a, 10b, 10c, 13I, 13p showed excellent activity against a panel of microorganisms. The newly synthesized compounds were characterized using IR, ${ }^{1} \mathrm{H}-\mathrm{NMR}$ and elemental analysis.
\end{abstract}

Keywords: Quinoline, chalcone, pyrimidine, antimicrobial activity

\section{Introduction}

In recent decades, problems of multi-drug resistant microorganisms have reached on alarming level in many countries around the world. A numbers of recent clinical reports describe the increasing occurrence of meticillin-resistant $S$. aureus and other antibiotic-resistant human pathogenic microorganisms in United State and European countries. Infections caused by those microorganisms pose a serious challenge to the medical community and the need for an effective therapy has led to a search for novel antimicrobial agents. In this work, we report the synthesis and biological activity of some quinolinyl chalcones and pyrimidines. Chalcones are a class of privileged structures that have a wide range of biological properties. ${ }^{1}$ Chalcones are also reported as anticancer agents, ${ }^{2}$ and antimalarial agents. ${ }^{3-6}$ Quinoline-based fused heterocyclic systems are found as potential anticancer agents ${ }^{7}$ and have antimalarial activities. ${ }^{8}$ Pyrimidine derivatives form a component in a number of useful drugs and are associated with many biological pharmaceutical and therapeutical activities. ${ }^{9}$ Condensed pyrimidine derivatives have been 
reported as analgesics, antiviral and as anti-inflammatory agents, ${ }^{10}$ antibacterial and antitubaculustic agents, ${ }^{11}$ diaryl pyrimidine (DAPY'S) appears to be the more effective against wild type and various mutant strains of HIV $-1 .{ }^{12}$

\section{Chemistry}

In this work, the main moiety, 2-chloro-3-formyl-7-methylquinoline 3 was synthesized using the Vilsmeier-Haack reagent according to the literature. ${ }^{13}$ In the ${ }^{1} \mathrm{H}-\mathrm{NMR}$ spectra of the chalcones, the protons of $\alpha \beta$ unsaturated system absorbed as two doublets around $\delta 7.5 \mathrm{ppm}$ for $\mathrm{H}-\alpha$ and 8.1 ppm for $\mathrm{H}-\beta$ with coupling constant $J=15-16 \mathrm{~Hz}$ for the trans isomer. Compound 4 was obtained in high yield and purity by condensation of $\mathbf{3}$ and morpholine in DMF. The product $\mathbf{4}$ was then treated with commercially available 4-aminoacetophenone to produce 5 which, on treatment with various $N$-chloroacetyl-4-(substitutedaryl)-6-(2,4-dichloro-5-fluoroaryl)pyrimidinyl amines, which were synthesized according to literature, ${ }^{14}$ gave 10a-g (Series 1) and compound 5 on treatment with various $N$-chloroacetyl arylamines in the presence of potassium carbonate in DMF gave 13h-q (Series 2). All the synthesized compounds were fully characterized by IR, ${ }^{1} \mathrm{H}-$ NMR spectroscopy and elemental analysis.
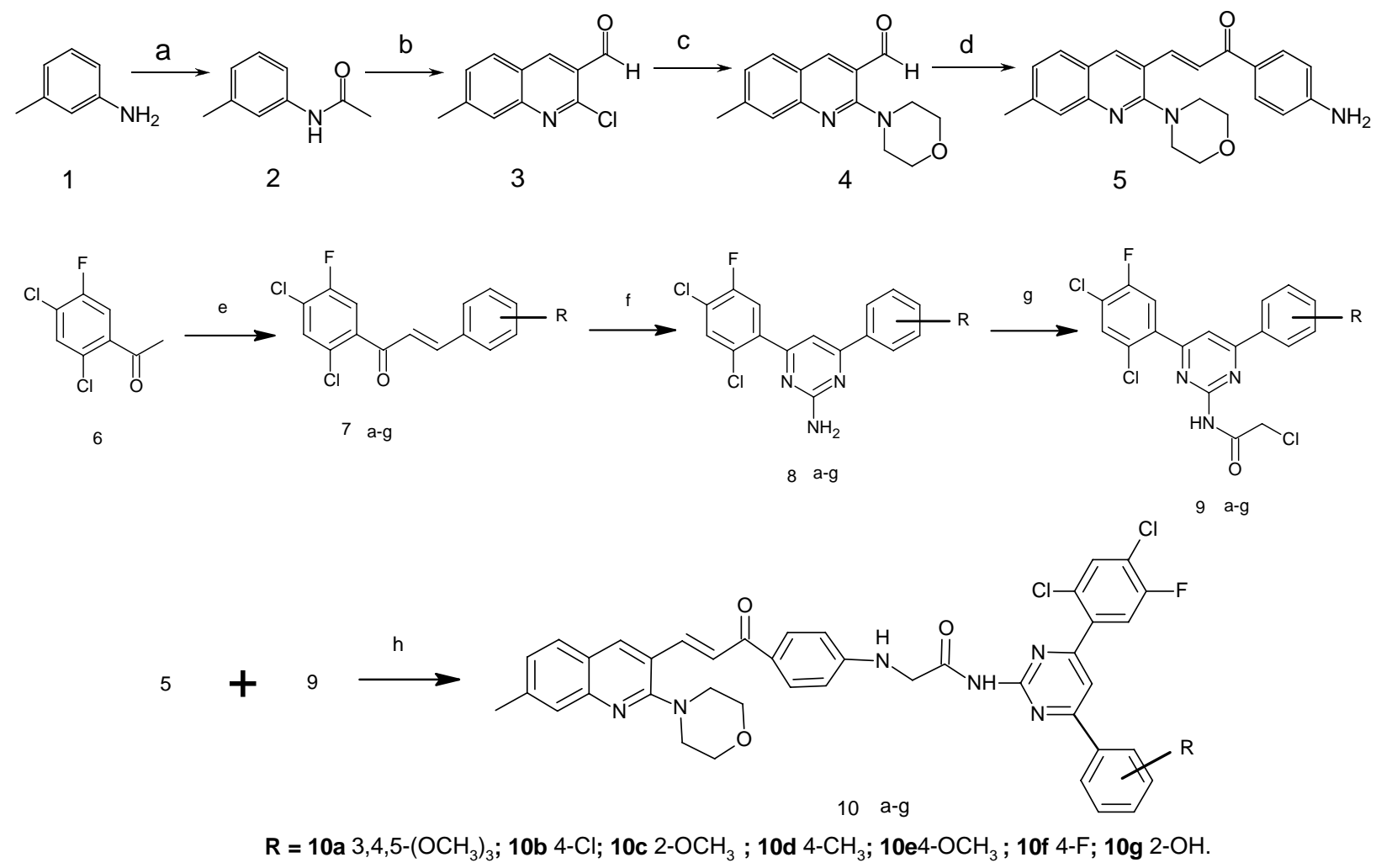

Series 1. Reagents: a) $\mathrm{Ac}_{2} \mathrm{O}, \mathrm{AcOH}$, reflux, 2 h. b) $\mathrm{DMF}, \mathrm{POCl}_{3}$ (3: 12), reflux, 6 h, 80-90 ${ }^{\circ} \mathrm{C}$. c) morpholine, DMF, $\mathrm{K}_{2} \mathrm{CO}_{3}$, reflux 3h. d) p-aminoacetophenone, $\mathrm{NaOMe}, \mathrm{MeOH}, 20 \% \mathrm{NaOH}$, stir, 24 h. e) Ar-CHO, NaOMe, $\mathrm{MeOH}, 20 \% \mathrm{NaOH}$, stir, 24 h. f) guanidine nitrate, $25 \% \mathrm{NaOMe}, \mathrm{MeOH}$, reflux temp, $7 \mathrm{hr}$. g) chloroacetyl chloride, benzene, TEA reflux at $90{ }^{\circ} \mathrm{C}, 3 \mathrm{~h}$. h) $\mathrm{K}_{2} \mathrm{CO}_{3}$, reflux, DMF, 5-10 h. 

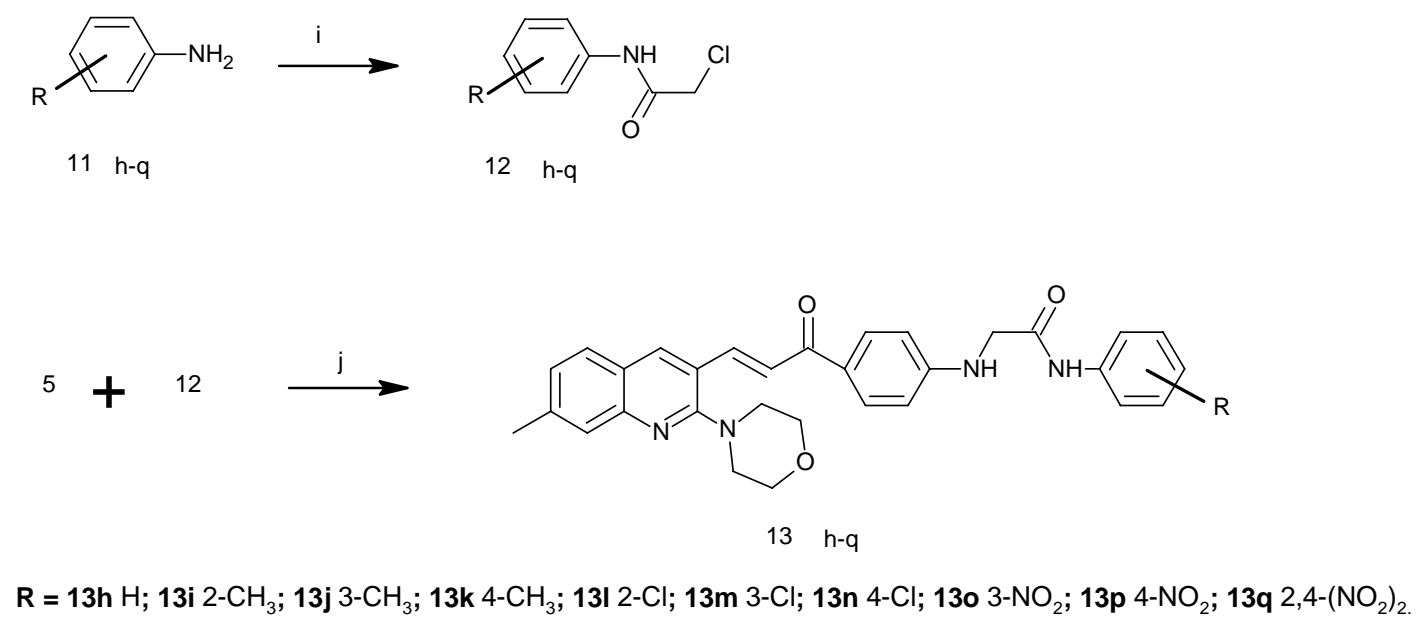

Series 2. Reagents: i) chloroacetyl chloride, benzene, TEA, reflux, $90{ }^{\circ} \mathrm{C}, 3 \mathrm{~h}$. j) $\mathrm{K}_{2} \mathrm{CO}_{3}$, reflux, DMF, $4 \mathrm{~h}$.

\section{Experimental Section}

General Procedures. All chemicals were of analytical grade and used directly. All melting points were determined in PMP-DM scientific melting point apparatus and are uncorrected. The completion of reaction was monitored by thin-layer chromatography (TLC) using silica gel-G coated Al-plates $(0.5 \mathrm{~mm}$ thickness, Merck) and spots were visualized under UV radiation. Infrared spectra were recorded on a Perkin Elmer RX-1 model spectrophotometer using $\mathrm{KBr}$ pellets. ${ }^{1} \mathrm{H}$ NMR spectra were acquired on a Bruker Avance-2 model spectrophotometer using $\mathrm{CDCl}_{3}$ as a solvent and TMS as internal reference (chemical shifts in $\delta$, ppm).

Preparation of 3-formyl-7-methyl-2-morpholinoquinoline (4). To a well-stirred solution of morpholine $(0.1 \mathrm{~mol}, 8.71 \mathrm{ml})$ and 2-chloro-3-formyl-7-methylquinoline $3(0.1 \mathrm{~mol}, 20.5 \mathrm{~g}$.) in DMF $(25 \mathrm{~mL})$ were stirred at $35{ }^{\circ} \mathrm{C}$ for $4 \mathrm{~h}$. The $\mathrm{pH}$ was adjusted using sodium bicarbonate. After cooling, the resulting solid was filtered, dried and recrystallized from ethanol to obtain compound 4 , yield $68 \%$, as yellowish white solid. Mp $178{ }^{\circ} \mathrm{C}$.

1-(4-aminophenyl)-3-(2-morpholino-7-methylquinolin-3-yl)-2-propen-1-one (5). To a solution of 4-aminoacetophenone (0.1 mol $13.5 \mathrm{~g}), 4(0.1 \mathrm{~mol}, 25.6 \mathrm{~g})$, methanol $(98 \mathrm{ml})$ and $20 \% \mathrm{NaOH}$ solution $(20 \mathrm{ml})$ was stirred for $0.5 \mathrm{~h}$ at room temperature and left overnight. After the completion of reaction, it was poured into ice water, acidified, filtered and recrystallized from ethanol to give a pale yellow powder. Yield $70 \%, \mathrm{Mp} 209^{\circ} \mathrm{C}$.

\section{Compound (10a). General method}

To a mixture of $5(0.01 \mathrm{~mol}, 3.73 \mathrm{~g}$. $)$ in $\mathrm{DMF}$ and the respectively $9 \mathrm{a}$ was heated at $90{ }^{\circ} \mathrm{C}$ in the presence of sodium bicarbonate for $5-10 \mathrm{~h}$ and the resulting mixture was poured into ice cold 
water and stirred for 30 minute then dry and washed with methanol and recrytallised from acrylonitrile. Yield 78\%, as a brown solid. Mp $189{ }^{\circ} \mathrm{C}$. Anal. Calcd for $\mathrm{C}_{44} \mathrm{H}_{39} \mathrm{~N}_{6} \mathrm{O}_{6} \mathrm{FCl}_{2}$ : C, 63.08\%; H, 4.69\%; N, 10.03\%. found: C, 63.05\%; H, 4.64\%; N, 10.01\%; NMR CDCl 2.38 (s, $3 \mathrm{H}, \mathrm{Me}), 3.69\left(\mathrm{t}, 4 \mathrm{H},-\mathrm{CH}_{2}\right), 3.60\left(\mathrm{t}, 4 \mathrm{H},-\mathrm{CH}_{2}\right), 4.00(\mathrm{~s}, 9 \mathrm{H}, \mathrm{OMe}), 4.05\left(\mathrm{~s}, 2 \mathrm{H},-\mathrm{CH}_{2}\right), 5.96(\mathrm{~s}$, $1 \mathrm{H},-\mathrm{NH}), 7.06\left(\mathrm{~s}, 1 \mathrm{H}, \mathrm{H}_{5}\right), 7.14\left(\mathrm{~s}, 1 \mathrm{H}, \mathrm{H}_{6}\right), 7.33\left(\mathrm{~s}, 1 \mathrm{H}, \mathrm{H}_{8}\right), 7.8(\mathrm{~s}, 1 \mathrm{H},-\mathrm{CH}), 7.88-8.52(\mathrm{~m}, 7 \mathrm{H}$, $\mathrm{Ar}-\mathrm{H}), 9.62$ (s, 1H, -NH), 8.1 (s, 1H, -CH). IR (KBr)/cm ${ }^{-1}: 3328 \mathrm{~cm}^{-1}(-\mathrm{NH}-), 1575 \mathrm{~cm}^{-1}(-\mathrm{C}=\mathrm{C}-$ ), $1635 \mathrm{~cm}^{-1}(-\mathrm{C}=\mathrm{N}-), 725 \mathrm{~cm}^{-1}(-\mathrm{C}-\mathrm{Cl}), 1098 \mathrm{~cm}^{-1}(-\mathrm{C}-\mathrm{F}-), 1684 \mathrm{~cm}^{-1}(>\mathrm{C}=\mathrm{O}-), 1130 \mathrm{~cm}^{-1}\left(-\mathrm{CH}_{2^{-}}\right.$ $\mathrm{O}-\mathrm{CH}_{2-}^{-}$.

Compound (10b). Yield 70\%, brown; mp 202; Anal. Calcd for $\mathrm{C}_{41} \mathrm{H}_{33} \mathrm{~N}_{6} \mathrm{O}_{3} \mathrm{FCl}_{2}$ : C, 62.96\%; $\mathrm{H}$, $4.12 \%$; N, 10.75\%. found: C, 62.93\%; H, 4.10\%; N, 10.73\%; NMR $\mathbf{C D C l}_{3}: 2.33$ (s, 3H, Me), $3.70\left(\mathrm{t}, 4 \mathrm{H},-\mathrm{CH}_{2}\right), 3.62\left(\mathrm{t}, 4 \mathrm{H},-\mathrm{CH}_{2}\right), 4.15\left(\mathrm{~s}, 2 \mathrm{H},-\mathrm{CH}_{2}\right), 5.98(\mathrm{~s}, 1 \mathrm{H},-\mathrm{NH}), 7.10\left(\mathrm{~s}, 1 \mathrm{H}, \mathrm{H}_{5}\right)$, $7.15\left(\mathrm{~s}, 1 \mathrm{H}, \mathrm{H}_{6}\right), 7.33\left(\mathrm{~s}, 1 \mathrm{H}, \mathrm{H}_{8}\right), 7.82(\mathrm{~s}, 1 \mathrm{H},-\mathrm{CH}), 7.88-8.52(\mathrm{~m}, 7 \mathrm{H}, \mathrm{Ar}-\mathrm{H}), 9.59(\mathrm{~s}, 1 \mathrm{H},-$ $\mathrm{NH}), 8.12(\mathrm{~s}, 1 \mathrm{H},-\mathrm{CH})$. IR (KBr)/cm ${ }^{-1}: 3330 \mathrm{~cm}^{-1}(-\mathrm{NH}-), 1560 \mathrm{~cm}^{-1}(-\mathrm{C}=\mathrm{C}-), 1648 \mathrm{~cm}^{-1}$ ($\mathrm{C}=\mathrm{N}-), 723 \mathrm{~cm}^{-1}$ (-C-Cl-), $1080 \mathrm{~cm}^{-1}$ (-C-F-), $1689 \mathrm{~cm}^{-1}(>\mathrm{C}=\mathrm{O}-), 1125 \mathrm{~cm}^{-1}\left(-\mathrm{CH}_{2}-\mathrm{O}-\mathrm{CH}_{2}-\right)$.

Compound (10c). Yield 62\%, brown; mp 165; Anal. Calcd for $\mathrm{C}_{42} \mathrm{H}_{35} \mathrm{~N}_{6} \mathrm{O}_{4} \mathrm{FCl}_{2}$ : C, 64.87\%; $\mathrm{H}$, 4.54\%; N, 10.81\%. found: C, 64.84\%; H, 4.51\%; N, 10.79\%; NMR $\mathbf{C D C l}_{3}: 2.37$ (s, 3H, Me), $3.66\left(\mathrm{t}, 4 \mathrm{H},-\mathrm{CH}_{2}\right), 3.59\left(\mathrm{t}, 4 \mathrm{H},-\mathrm{CH}_{2}\right), 4.01(\mathrm{~s}, 3 \mathrm{H}, \mathrm{OMe}), 4.06\left(\mathrm{~s}, 2 \mathrm{H},-\mathrm{CH}_{2}\right), 5.96(\mathrm{~s}, 1 \mathrm{H},-\mathrm{NH})$, $7.06\left(\mathrm{~s}, 1 \mathrm{H}, \mathrm{H}_{5}\right), 7.14\left(\mathrm{~s}, 1 \mathrm{H}, \mathrm{H}_{6}\right), 7.33\left(\mathrm{~s}, 1 \mathrm{H}, \mathrm{H}_{8}\right), 7.8(\mathrm{~s}, 1 \mathrm{H},-\mathrm{CH}), 7.33\left(\mathrm{~s}, 1 \mathrm{H}, \mathrm{H}_{8}\right), 7.88-8.52$ (m, 7H, Ar-H), 9.62 (s, 1H, -NH), 8.1 (s, 1H, -CH). IR (KBr)/cm ${ }^{-1}: 3340 \mathrm{~cm}^{-1}(-\mathrm{NH}-), 1580 \mathrm{~cm}^{-1}$ $(-\mathrm{C}=\mathrm{C}-), 1645 \mathrm{~cm}^{-1}(-\mathrm{C}=\mathrm{N}-), 730 \mathrm{~cm}^{-1}(-\mathrm{C}-\mathrm{Cl}-), 1090 \mathrm{~cm}^{-1}(-\mathrm{C}-\mathrm{F}-), 1684 \mathrm{~cm}^{-1}(>\mathrm{C}=\mathrm{O}-), 1140 \mathrm{~cm}^{-}$ ${ }^{1}\left(-\mathrm{CH}_{2}-\mathrm{O}-\mathrm{CH}_{2}-\right)$.

Compound (10d). Yield 70\%, brown; mp 168; Anal. Calcd for $\mathrm{C}_{42} \mathrm{H}_{35} \mathrm{~N}_{6} \mathrm{O}_{3} \mathrm{FCl}_{2}$ : $\mathrm{C}, 66.23 \%$; $\mathrm{H}$, 4.63\%; N, 11.03\%. found: C, 66.21\%; H, 4.62\%; N, 10.01\%; $\mathbf{N M R}_{\mathbf{C D C l}}$ : 2.32 (s, 3H, Me), $2.38(\mathrm{~s}, 3 \mathrm{H}, \mathrm{Me}), 3.69\left(\mathrm{t}, 4 \mathrm{H},-\mathrm{CH}_{2}\right), 3.60\left(\mathrm{~s}, 4 \mathrm{H},-\mathrm{CH}_{2}\right), 4.08\left(\mathrm{~s}, 2 \mathrm{H},-\mathrm{CH}_{2}\right), 5.92(\mathrm{~s}, 1 \mathrm{H},-\mathrm{NH})$, $7.08\left(\mathrm{~s}, 1 \mathrm{H}, \mathrm{H}_{5}\right), 7.15\left(\mathrm{~s}, 1 \mathrm{H}, \mathrm{H}_{6}\right), 7.30\left(\mathrm{~s}, 1 \mathrm{H}, \mathrm{H}_{8}\right), 7.86(\mathrm{~s}, 1 \mathrm{H},-\mathrm{CH}), 7.34\left(\mathrm{~s}, 1 \mathrm{H}, \mathrm{H}_{8}\right), 7.88-8.52$ (m, 7H, Ar-H), 9.58 (s, 1H, -NH), 8.12 (s, 1H, -CH). IR (KBr)/cm ${ }^{-1}: 3330$ cm $^{-1}(-\mathrm{NH}-), 1575$ $\mathrm{cm}^{-1}(-\mathrm{C}=\mathrm{C}-), 1635 \mathrm{~cm}^{-1}(-\mathrm{C}=\mathrm{N}-), 720 \mathrm{~cm}^{-1}(-\mathrm{C}-\mathrm{Cl}-), 1098 \mathrm{~cm}^{-1}$ (-C-F-), $1678 \mathrm{~cm}^{-1}(>\mathrm{C}=\mathrm{O}-), 1150$ $\mathrm{cm}^{-1}\left(-\mathrm{CH}_{2}-\mathrm{O}-\mathrm{CH}_{2}-\right)$.

Compound (10e). Yield 65\%, brown; mp 194; Anal. Calcd for $\mathrm{C}_{42} \mathrm{H}_{35} \mathrm{~N}_{6} \mathrm{O}_{4} \mathrm{FCl}_{2} \mathrm{C}, 64.87 \%$; $\mathrm{H}$, 4.54\%; N, 10.81\%. found: C, 64.85\%; H, 4.50\%; N, 10.78\%; $\mathbf{N M R} \mathbf{C D C l}_{3}: 2.36$ (s, 3H, Me), $3.66\left(\mathrm{t}, 4 \mathrm{H},-\mathrm{CH}_{2}\right), 3.59\left(\mathrm{t}, 4 \mathrm{H},-\mathrm{CH}_{2}\right), 4.01(\mathrm{~s}, 3 \mathrm{H}, \mathrm{OMe}), 4.09\left(\mathrm{~s}, 2 \mathrm{H},-\mathrm{CH}_{2}\right), 5.92(\mathrm{~s}, 1 \mathrm{H},-\mathrm{NH})$, $7.09\left(\mathrm{~s}, 1 \mathrm{H}, \mathrm{H}_{5}\right), 7.15\left(\mathrm{~s}, 1 \mathrm{H}, \mathrm{H}_{6}\right), 7.33\left(\mathrm{~s}, 1 \mathrm{H}, \mathrm{H}_{8}\right), 7.8(\mathrm{~s}, 1 \mathrm{H},-\mathrm{CH}), 7.33\left(\mathrm{~s}, 1 \mathrm{H}, \mathrm{H}_{8}\right), 7.88-8.52$ (m, 7H, Ar-H), 9.62 (s, 1H, -NH), 8.1 (s, 1H, -CH). IR (KBr)/cm ${ }^{-1}: 3320$ cm$^{-1}(-\mathrm{NH}-), 1566 \mathrm{~cm}^{-}$ ${ }^{1}(-\mathrm{C}=\mathrm{C}-), 1630 \mathrm{~cm}^{-1}(-\mathrm{C}=\mathrm{N}-), 732 \mathrm{~cm}^{-1}(-\mathrm{C}-\mathrm{Cl}-), 1088 \mathrm{~cm}^{-1}(-\mathrm{C}-\mathrm{F}-), 1673 \mathrm{~cm}^{-1}(>\mathrm{C}=\mathrm{O}-), 1150 \mathrm{~cm}^{-}$ ${ }^{1}\left(-\mathrm{CH}_{2}-\mathrm{O}-\mathrm{CH}_{2}-\right)$.

Compound (10f). Yield 70\%, brown; mp 210; Anal. Calcd for $\mathrm{C}_{41} \mathrm{H}_{33} \mathrm{~N}_{6} \mathrm{O}_{3} \mathrm{~F}_{2} \mathrm{Cl}_{2}$ : $\mathrm{C}, 62.96 \%$; $\mathrm{H}$, 4.12\%; N, 10.75\%. found: C, 62.94\%; H, 4.09\%; N, 10.73\%; $\mathbf{N M R}_{\mathbf{C D C l}}$ : 2.36 (s, 3H, Me), $3.66\left(\mathrm{t}, 4 \mathrm{H},-\mathrm{CH}_{2}\right), 3.59\left(\mathrm{t}, 4 \mathrm{H},-\mathrm{CH}_{2}\right), 4.12\left(\mathrm{~s}, 2 \mathrm{H},-\mathrm{CH}_{2}\right), 6.01(\mathrm{~s}, 1 \mathrm{H},-\mathrm{NH}), 7.12\left(\mathrm{~s}, 1 \mathrm{H}, \mathrm{H}_{5}\right)$, $7.19\left(\mathrm{~s}, 1 \mathrm{H}, \mathrm{H}_{6}\right), 7.38\left(\mathrm{~s}, 1 \mathrm{H}, \mathrm{H}_{8}\right), 7.8(\mathrm{~s}, 1 \mathrm{H},-\mathrm{CH}), 7.33\left(\mathrm{~s}, 1 \mathrm{H}, \mathrm{H}_{8}\right), 7.88-8.52(\mathrm{~m}, 7 \mathrm{H}, \operatorname{Ar}-\mathrm{H})$, 9.67 (s, 1H, -NH), 8.12 (s, 1H, -CH). IR (KBr)/cm ${ }^{-1}: 3327$ cm$^{-1}(-\mathrm{NH}-), 1576 \mathrm{~cm}^{-1}(-\mathrm{C}=\mathrm{C}-)$, 
$1655 \mathrm{~cm}^{-1}(-\mathrm{C}=\mathrm{N}-), 721 \mathrm{~cm}^{-1}(-\mathrm{C}-\mathrm{Cl}-), 1065 \mathrm{~cm}^{-1}(-\mathrm{C}-\mathrm{F}-), 1679 \mathrm{~cm}^{-1}(>\mathrm{C}=\mathrm{O}-), 1146 \mathrm{~cm}^{-1}\left(-\mathrm{CH}_{2}-\mathrm{O}-\right.$ $\mathrm{CH}_{2-}$ ).

Compound (10g). Yield 60\%, brown; mp 155; Anal. Calcd for $\mathrm{C}_{41} \mathrm{H}_{33} \mathrm{~N}_{6} \mathrm{O}_{4} \mathrm{FCl}_{2}: \mathrm{C}, 62.49 \%$; $\mathrm{H}$, 4.36\%; N, 11.01\%. found: C, 62.47\%; H, 4.33\%; N, 10.98\%; NMR CDCl $: 2.33$ (s, 3H, Me), $3.68\left(\mathrm{t}, 4 \mathrm{H},-\mathrm{CH}_{2}\right), 3.58\left(\mathrm{t}, 4 \mathrm{H},-\mathrm{CH}_{2}\right), 4.08\left(\mathrm{~s}, 2 \mathrm{H},-\mathrm{CH}_{2}\right), 6.01(\mathrm{~s}, 1 \mathrm{H},-\mathrm{NH}), 7.14\left(\mathrm{~s}, 1 \mathrm{H}, \mathrm{H}_{5}\right)$, $7.11\left(\mathrm{~s}, 1 \mathrm{H}, \mathrm{H}_{6}\right), 7.35\left(\mathrm{~s}, 1 \mathrm{H}, \mathrm{H}_{8}\right), 7.82(\mathrm{~s}, 1 \mathrm{H},-\mathrm{CH}), 7.32\left(\mathrm{~s}, 1 \mathrm{H}, \mathrm{H}_{8}\right), 7.88-8.52(\mathrm{~m}, 7 \mathrm{H}, \mathrm{Ar}-\mathrm{H})$, 9.65 (s, $1 \mathrm{H},-\mathrm{NH}), 8.18(\mathrm{~s}, 1 \mathrm{H},-\mathrm{CH})$. IR (KBr)/ $\mathrm{cm}^{-1}: 3332 \mathrm{~cm}^{-1}(-\mathrm{NH}-), 1576 \mathrm{~cm}^{-1}(-\mathrm{C}=\mathrm{C}-)$, $1638 \mathrm{~cm}^{-1}(-\mathrm{C}=\mathrm{N}-), 712 \mathrm{~cm}^{-1}(-\mathrm{C}-\mathrm{Cl}-), 1092 \mathrm{~cm}^{-1}(-\mathrm{C}-\mathrm{F}-), 1663 \mathrm{~cm}^{-1}(>\mathrm{C}=\mathrm{O}-), 1159 \mathrm{~cm}^{-1}\left(-\mathrm{CH}_{2}-\mathrm{O}-\right.$ $\left.\mathrm{CH}_{2-}\right)$.

\section{General procedure for $\boldsymbol{N}$-chloroacetyl arylamines $(\mathbf{1 2 h}-\mathbf{q})$}

In benzene $(30 \mathrm{ml})$, chloroacetyl chloride $(0.03 \mathrm{~mol}, 3.38 \mathrm{~g}, 2.4 \mathrm{ml})$ and 2-3 drops of TEA were added and the mixture was stirred on a water bath for 10 mins. A solution of arylamine $(0.02$ mol) in benzene $(30 \mathrm{ml})$ was added dropwise and the mixture heated at reflux for $2 \mathrm{~h}$ then cooled. The resulting white precipitate was filtered and washed with benzene, purified by recrystallization from alcohol.

Compound (13h). Compound 5 (0.01 mol, 3.73 g.) and the $N$-chloroacetyl aryl amine 12h were heated at reflux in DMF $(25 \mathrm{ml})$ at $90{ }^{\circ} \mathrm{C}$ in the presence of sodium bicarbonate for $5-10 \mathrm{~h}$ and the resulting mixrture was poured into ice cold water and stirred for 30 min then dry and washed with methanol and recrytallisation form acrylonitrile or ethanol, yield $65 \%$, brown solid. $\mathrm{Mp}$ 220-225 ${ }^{\circ}$ C. Anal. Calcd for $\mathrm{C}_{31} \mathrm{H}_{30} \mathrm{~N}_{4} \mathrm{O}_{3}: \mathrm{C}, 73.44 \%$; H, 5.92\%; N, $11.05 \%$. found: $\mathrm{C}, 73.41 \%$; H, 5.89\%; N, 11.02\%; NMR CDCl 2.40 (s, 3H, Me), $9.64(\mathrm{~s}, 1 \mathrm{H},-\mathrm{NH}), 5.97$ (s, 1H, -NH), $4.08\left(\mathrm{~s}, 2 \mathrm{H},-\mathrm{CH}_{2}\right), 7.61(\mathrm{~m}, 4 \mathrm{H}, \mathrm{Ar}-\mathrm{H}), 7.24(\mathrm{~m}, 4 \mathrm{H}, \mathrm{Ar}-\mathrm{H}), 3.71\left(\mathrm{t}, 4 \mathrm{H},-\mathrm{CH}_{2}\right), 3.62(\mathrm{t}, 4 \mathrm{H},-$ $\left.\mathrm{CH}_{2}\right), 8.12(\mathrm{~d}, 1 \mathrm{H},-\mathrm{CH}), 7.5(\mathrm{~d}, 1 \mathrm{H},-\mathrm{CH}), 7.12-8.28(\mathrm{~m}, 4 \mathrm{H}, \mathrm{Ar}-\mathrm{H}) . \mathbf{I R}(\mathbf{K B r}) / \mathrm{cm}^{-1}: 3336 \mathrm{~cm}^{-}$ ${ }^{1}(-\mathrm{NH}-), 1580 \mathrm{~cm}^{-1}(>\mathrm{C}=\mathrm{O}), 1638 \mathrm{~cm}^{-1}(-\mathrm{C}=\mathrm{N}-), 1335 \mathrm{~cm}^{-1}\left(-\mathrm{C}_{-} \mathrm{CH}_{3}\right), 1111 \mathrm{~cm}^{-1}\left(-\mathrm{CH}_{2}-\mathrm{O}_{-} \mathrm{CH}_{2}-\right)$.

Compound (13i). Yield 71\%, brown; mp 230; Anal. Calcd for $\mathrm{C}_{32} \mathrm{H}_{32} \mathrm{~N}_{4} \mathrm{O}_{3}: \mathrm{C}, 73.84 \%$; $\mathrm{H}$, 6.14\%; N, 10.75\%. found: C, 73.81\%; H, 6.12\%; N, 10.73\%; NMR $\mathbf{C D C l}_{3}: 2.38$ (s, 3H, Me), $2.42(\mathrm{~s}, 3 \mathrm{H}, \mathrm{Me}), 9.64(\mathrm{~s}, 1 \mathrm{H},-\mathrm{NH}), 6.07(\mathrm{~s}, 1 \mathrm{H},-\mathrm{NH}), 4.05\left(\mathrm{~s}, 2 \mathrm{H},-\mathrm{CH}_{2}\right), 7.58(\mathrm{~m}, 4 \mathrm{H}, \mathrm{Ar}-\mathrm{H})$, $7.21(\mathrm{~m}, 4 \mathrm{H}, \mathrm{Ar}-\mathrm{H}), 3.79$ (t, 4H, $\left.-\mathrm{CH}_{2}\right), 3.68\left(\mathrm{t}, 4 \mathrm{H},-\mathrm{CH}_{2}\right), 8.10(\mathrm{~d}, 1 \mathrm{H},-\mathrm{CH}), 7.49(\mathrm{~d}, 1 \mathrm{H},-\mathrm{CH})$, 7.12-8.28 (m, 4H, Ar-H). IR (KBr)/cm ${ }^{-1}: 3342 \mathrm{~cm}^{-1}(-\mathrm{NH}-), 1588 \mathrm{~cm}^{-1}(>\mathrm{C}=\mathrm{O}), 1635 \mathrm{~cm}^{-1}(-$ $\mathrm{C}=\mathrm{N}-), 1343 \mathrm{~cm}^{-1}\left(-\mathrm{C}-\mathrm{CH}_{3}\right), 1123 \mathrm{~cm}^{-1}\left(-\mathrm{CH}_{2}-\mathrm{O}-\mathrm{CH}_{2}-\right)$.

Compound (13j). Yield 77\%, brwon; mp 228; Anal. Calcd for $\mathrm{C}_{32} \mathrm{H}_{32} \mathrm{~N}_{4} \mathrm{O}_{3}: \mathrm{C}, 73.84 \%$; $\mathrm{H}$, 6.14\%; N, 10.75\%. found: C, 73.82\%; H, 6.11\%; N, 10.72\%; NMR $\mathbf{C D C l}_{3}: 2.38$ (s, 3H, Me), $2.33(\mathrm{~s}, 3 \mathrm{H}, \mathrm{Me}), 9.66(\mathrm{~s}, 1 \mathrm{H},-\mathrm{NH}), 5.88(\mathrm{~s}, 1 \mathrm{H},-\mathrm{NH}), 4.01\left(\mathrm{~s}, 2 \mathrm{H},-\mathrm{CH}_{2}\right), 7.51(\mathrm{~m}, 4 \mathrm{H}, \mathrm{Ar}-\mathrm{H})$, $7.26(\mathrm{~m}, 4 \mathrm{H}, \mathrm{Ar}-\mathrm{H}), 3.83\left(\mathrm{t}, 4 \mathrm{H},-\mathrm{CH}_{2}\right), 3.62\left(\mathrm{t}, 4 \mathrm{H},-\mathrm{CH}_{2}\right), 8.10(\mathrm{~d}, 1 \mathrm{H},-\mathrm{CH}), 7.49(\mathrm{~d}, 1 \mathrm{H},-\mathrm{CH})$, 7.12-8.28 (m, 4H, Ar-H). IR (KBr)/cm ${ }^{-1}: 3348 \mathrm{~cm}^{-1}(-\mathrm{NH}-), 1581 \mathrm{~cm}^{-1}(>\mathrm{C}=\mathrm{O}), 1631 \mathrm{~cm}^{-1}(-$ $\mathrm{C}=\mathrm{N}-), 1347 \mathrm{~cm}^{-1}\left(-\mathrm{C}-\mathrm{CH}_{3}\right), 1121 \mathrm{~cm}^{-1}\left(-\mathrm{CH}_{2}-\mathrm{O}-\mathrm{CH}_{2}-\right)$.

Compound (13k). Yield 55\%, brwon; mp 222; Anal. Calcd for $\mathrm{C}_{32} \mathrm{H}_{32} \mathrm{~N}_{4} \mathrm{O}_{3}: \mathrm{C}, 73.84 \%$; $\mathrm{H}$, 6.14\%; N, 10.75\%. found: C, 73.83\%; H, 6.10\%; N, 10.73\%; NMR $\mathbf{C D C l}_{3}: 2.35$ (s, 3H, Me), 2.47 (s, 3H, Me), $9.63(\mathrm{~s}, 1 \mathrm{H},-\mathrm{NH}), 6.12(\mathrm{~s}, 1 \mathrm{H},-\mathrm{NH}), 4.09$ (s, 2H, - $\left.\mathrm{CH}_{2}\right), 7.5$ (m, 4H, Ar-H), 
$7.24(\mathrm{~m}, 4 \mathrm{H}, \mathrm{Ar}-\mathrm{H}), 3.80\left(\mathrm{t}, 4 \mathrm{H},-\mathrm{CH}_{2}\right), 3.68\left(\mathrm{t}, 4 \mathrm{H},-\mathrm{CH}_{2}\right), 8.10(\mathrm{~d}, 1 \mathrm{H},-\mathrm{CH}), 7.54(\mathrm{~d}, 1 \mathrm{H},-\mathrm{CH})$, 7.12-8.28 (m, 4H, Ar-H). IR (KBr)/cm ${ }^{-1}: 3328 \mathrm{~cm}^{-1}(-\mathrm{NH}-), 1557 \mathrm{~cm}^{-1}(>\mathrm{C}=\mathrm{O}), 1649 \mathrm{~cm}^{-1}(-$ $\mathrm{C}=\mathrm{N}-), 1325 \mathrm{~cm}^{-1}\left(-\mathrm{C}-\mathrm{CH}_{3}\right), 1108 \mathrm{~cm}^{-1}\left(-\mathrm{CH}_{2}-\mathrm{O}-\mathrm{CH}_{2}-\right)$.

Compound (13l). Yield 65\%, brwon; mp 165; Anal. Calcd for $\mathrm{C}_{32} \mathrm{H}_{29} \mathrm{~N}_{4} \mathrm{O}_{3} \mathrm{Cl}$ : C, 67.76\%; $\mathrm{H}$, 5.36\%; N, 10.35\%. found: C, 67.74\%; H, 5.34\%; N, 10.33\%; NMR $\mathbf{C D C l}_{3}: 2.38$ (s, 3H, Me), $9.60(\mathrm{~s}, 1 \mathrm{H},-\mathrm{NH}), 5.96(\mathrm{~s}, 1 \mathrm{H},-\mathrm{NH}), 4.13\left(\mathrm{~s}, 2 \mathrm{H},-\mathrm{CH}_{2}\right), 7.58(\mathrm{~m}, 4 \mathrm{H}, \mathrm{Ar}-\mathrm{H}), 7.21$ (m, 4H, Ar$\mathrm{H}), 3.79\left(\mathrm{t}, 4 \mathrm{H},-\mathrm{CH}_{2}\right), 3.64\left(\mathrm{t}, 4 \mathrm{H},-\mathrm{CH}_{2}\right), 8.10(\mathrm{~d}, 1 \mathrm{H},-\mathrm{CH}), 7.49(\mathrm{~d}, 1 \mathrm{H},-\mathrm{CH}), 7.12-8.28(\mathrm{~m}$, $4 \mathrm{H}, \mathrm{Ar}-\mathrm{H})$. IR (KBr)/cm ${ }^{-1}: 3342 \mathrm{~cm}^{-1}(-\mathrm{NH}-), 1588 \mathrm{~cm}^{-1}(>\mathrm{C}=\mathrm{O}), 1635 \mathrm{~cm}^{-1}(-\mathrm{C}=\mathrm{N}-), 1343 \mathrm{~cm}^{-}$ ${ }^{1}\left(-\mathrm{C}-\mathrm{CH}_{3}\right), 1123 \mathrm{~cm}^{-1}\left(-\mathrm{CH}_{2}-\mathrm{O}-\mathrm{CH}_{2}-\right), 711 \mathrm{~cm}^{-1}(-\mathrm{C}-\mathrm{Cl}-)$.

Compound (13m). Yield 73\%, brwon; mp 218; Anal. Calcd for $\mathrm{C}_{31} \mathrm{H}_{29} \mathrm{~N}_{4} \mathrm{O}_{3} \mathrm{Cl}$ : C, 67.76\%; $\mathrm{H}$, $5.36 \%$; N, 10.35\%. found: C, 67.73\%; H, 5.32\%; N, 10.32\%; NMR $\mathbf{C D C l}_{3}: 2.38$ (s, 3H, Me), $9.56(\mathrm{~s}, 1 \mathrm{H},-\mathrm{NH}), 6.07$ (s, 1H, -NH), $3.92\left(\mathrm{~s}, 2 \mathrm{H},-\mathrm{CH}_{2}\right), 7.58(\mathrm{~m}, 4 \mathrm{H}, \mathrm{Ar}-\mathrm{H}), 7.18$ (m, 4H, Ar$\mathrm{H}), 3.85$ (t, 4H, $\left.-\mathrm{CH}_{2}\right), 3.68\left(\mathrm{t}, 4 \mathrm{H},-\mathrm{CH}_{2}\right), 8.10(\mathrm{~d}, 1 \mathrm{H},-\mathrm{CH}), 7.49(\mathrm{~d}, 1 \mathrm{H},-\mathrm{CH}), 7.12-8.28(\mathrm{~m}$, $4 \mathrm{H}, \mathrm{Ar}-\mathrm{H})$. IR (KBr)/cm ${ }^{-1}: 3322 \mathrm{~cm}^{-1}(-\mathrm{NH}-), 1578 \mathrm{~cm}^{-1}(>\mathrm{C}=\mathrm{O}), 1631 \mathrm{~cm}^{-1}(-\mathrm{C}=\mathrm{N}-), 1323 \mathrm{~cm}^{-}$ ${ }^{1}\left(-\mathrm{C}-\mathrm{CH}_{3}\right), 1117 \mathrm{~cm}^{-1}\left(-\mathrm{CH}_{2}-\mathrm{O}-\mathrm{CH}_{2}-\right), 718 \mathrm{~cm}^{-1}(-\mathrm{C}-\mathrm{Cl}-)$.

Compound (13n). Yield 78\%, brwon; mp 249; Anal. Calcd for $\mathrm{C}_{31} \mathrm{H}_{29} \mathrm{~N}_{4} \mathrm{O}_{3} \mathrm{Cl}: \mathrm{C}, 67.76 \%$; $\mathrm{H}$, 5.36\%; N, 10.35\%. found: C, 67.73\%; H, 5.34\%; N, 10.31\%; NMR $\mathbf{C D C l}_{3}: 2.34(\mathrm{~s}, 3 \mathrm{H}, \mathbf{M e})$, 9.52(s, 1H, -NH), 6.02(s, 1H, -NH), 3.97 (s, 2H, - $\left.\mathrm{CH}_{2}\right), 7.55$ (m, 4H, Ar-H), 7.13 (m, 4H, Ar-H), $3.83\left(\mathrm{t}, 4 \mathrm{H},-\mathrm{CH}_{2}\right), 3.64\left(\mathrm{t}, 4 \mathrm{H},-\mathrm{CH}_{2}\right), 8.10(\mathrm{~d}, 1 \mathrm{H},-\mathrm{CH}), 7.44(\mathrm{~d}, 1 \mathrm{H},-\mathrm{CH}), 7.12-8.28(\mathrm{~m}, 4 \mathrm{H}$, $\mathrm{Ar}-\mathrm{H})$. IR (KBr)/cm ${ }^{-1}: 3352 \mathrm{~cm}^{-1}(-\mathrm{NH}-), 1598 \mathrm{~cm}^{-1}(>\mathrm{C}=\mathrm{O}), 1643 \mathrm{~cm}^{-1}(-\mathrm{C}=\mathrm{N}-), 1343 \mathrm{~cm}^{-1}(-\mathrm{C}-$ $\left.\mathrm{CH}_{3}\right), 1133 \mathrm{~cm}^{-1}\left(-\mathrm{CH}_{2}-\mathrm{O}-\mathrm{CH}_{2}-\right), 704 \mathrm{~cm}^{-1}(-\mathrm{C}-\mathrm{Cl}-)$.

Compound (13o). Yield 80\%, brwon; mp 257; Anal. Calcd for $\mathrm{C}_{31} \mathrm{H}_{29} \mathrm{~N}_{5} \mathrm{O}_{5}$ : C, 67.45\%; $\mathrm{H}$, 5.25\%; N, 12.69\%. found: C, 67.41\%; H, 5.22\%; N, 12.67\%; NMR $\mathbf{C D C l}_{3}: 2.42$ (s, 3H, Me), $9.64(\mathrm{~s}, 1 \mathrm{H},-\mathrm{NH}), 6.03(\mathrm{~s}, 1 \mathrm{H},-\mathrm{NH}), 4.01\left(\mathrm{~s}, 2 \mathrm{H},-\mathrm{CH}_{2}\right), 7.58(\mathrm{~m}, 4 \mathrm{H}, \mathrm{Ar}-\mathrm{H}), 7.21$ (m, 4H, Ar$\mathrm{H}), 3.79$ (t, 4H, $\left.-\mathrm{CH}_{2}\right), 3.64\left(\mathrm{t}, 4 \mathrm{H},-\mathrm{CH}_{2}\right), 8.10(\mathrm{~d}, 1 \mathrm{H},-\mathrm{CH}), 7.47(\mathrm{~d}, 1 \mathrm{H},-\mathrm{CH}), 7.12-8.28(\mathrm{~m}$, $4 \mathrm{H}, \mathrm{Ar}-\mathrm{H})$. IR (KBr)/cm ${ }^{-1}: 3352 \mathrm{~cm}^{-1}(-\mathrm{NH}-), 1572 \mathrm{~cm}^{-1}(>\mathrm{C}=\mathrm{O}), 1645 \mathrm{~cm}^{-1}(-\mathrm{C}=\mathrm{N}-), 1339 \mathrm{~cm}^{-}$ ${ }^{1}\left(-\mathrm{C}-\mathrm{CH}_{3}\right), 1126 \mathrm{~cm}^{-1}\left(-\mathrm{CH}_{2}-\mathrm{O}-\mathrm{CH}_{2}-\right)$.

Compound (13p). Yield 76\%, brwon; mp 232; Anal. Calcd for $\mathrm{C}_{31} \mathrm{H}_{29} \mathrm{~N}_{5} \mathrm{O}_{5}$ : C, 67.45\%; $\mathrm{H}$, 5.25\%; N, 12.69\%. found: C, 67.42\%; H, 5.21\%; N, 12.67\%; NMR $\mathbf{C D C l}_{3}: 2.33$ (s, 3H, Me), 9.67 (s, 1H, -NH), 6.07 (s, 1H, -NH), 4.05 (s, 2H, -CH $), 7.58$ (m, 4H, Ar-H), 7.21 (m, 4H, Ar$\mathrm{H}), 3.72\left(\mathrm{t}, 4 \mathrm{H},-\mathrm{CH}_{2}\right), 3.68\left(\mathrm{t}, 4 \mathrm{H},-\mathrm{CH}_{2}\right), 8.10(\mathrm{~d}, 1 \mathrm{H},-\mathrm{CH}), 7.49(\mathrm{~d}, 1 \mathrm{H},-\mathrm{CH}), 7.12-8.28(\mathrm{~m}$, $4 \mathrm{H}, \mathrm{Ar}-\mathrm{H})$. IR (KBr)/cm ${ }^{-1}: 3332 \mathrm{~cm}^{-1}(-\mathrm{NH}-), 1578 \mathrm{~cm}^{-1}(>\mathrm{C}=\mathrm{O}), 1652 \mathrm{~cm}^{-1}(-\mathrm{C}=\mathrm{N}-), 1345 \mathrm{~cm}^{-}$ ${ }^{1}\left(-\mathrm{C}-\mathrm{CH}_{3}\right), 1123 \mathrm{~cm}^{-1}\left(-\mathrm{CH}_{2}-\mathrm{O}-\mathrm{CH}_{2}-\right)$.

Compound (13q). Yield 69\%, brwon; mp 242; Anal. Calcd for $\mathrm{C}_{31} \mathrm{H}_{28} \mathrm{~N}_{6} \mathrm{O}_{7}$ : C, 72.75\%; $\mathrm{H}$, 4.69\%; N, 14.08\%. found: C, 72.71\%; H, 4.67\%; N, 14.06\%; NMR $\mathbf{C D C l}_{3}: 2.42$ (s, 3H, Me), $9.69(\mathrm{~s}, 1 \mathrm{H},-\mathrm{NH}), 6.07(\mathrm{~s}, 1 \mathrm{H},-\mathrm{NH}), 4.02\left(\mathrm{~s}, 2 \mathrm{H},-\mathrm{CH}_{2}\right), 7.54(\mathrm{~m}, 4 \mathrm{H}, \mathrm{Ar}-\mathrm{H}), 7.21$ (m, 4H, Ar$\mathrm{H}), 3.74\left(\mathrm{t}, 4 \mathrm{H},-\mathrm{CH}_{2}\right), 3.68\left(\mathrm{t}, 4 \mathrm{H},-\mathrm{CH}_{2}\right), 8.10(\mathrm{~d}, 1 \mathrm{H},-\mathrm{CH}), 7.42(\mathrm{~d}, 1 \mathrm{H},-\mathrm{CH}), 7.12-8.28(\mathrm{~m}$, $5 \mathrm{H}, \mathrm{Ar}-\mathrm{H})$. IR (KBr)/cm ${ }^{-1}: 3344 \mathrm{~cm}^{-1}(-\mathrm{NH}-), 1578 \mathrm{~cm}^{-1}(>\mathrm{C}=\mathrm{O}), 1635 \mathrm{~cm}^{-1}(-\mathrm{C}=\mathrm{N}-), 1341 \mathrm{~cm}^{-}$ ${ }^{1}\left(-\mathrm{C}-\mathrm{CH}_{3}\right), 1112 \mathrm{~cm}^{-1}\left(-\mathrm{CH}_{2}-\mathrm{O}-\mathrm{CH}_{2}-\right)$. 


\section{Biological assays}

\section{Compounds}

Test compounds were dissolved in DMSO (12.5\%) at an initial concentration of $20 \mathrm{mg} \mathrm{ml}^{-1}$ and then were serially diluted in culture medium.

\section{Cells}

Bacterial strains Staphylococcus aureus, Bacillus subtillis, Escherichia coli, Salmonella typhosa.

\section{Antibacterial assays}

The MICs of the chemical compounds assays were carried out as described by Clause ${ }^{15}$ with minor modifications. Ampicillin trihydrate was used as reference antibacterial agent. Solutions of the test compounds and reference drug were dissolved in DMSO at a concentration of $20 \mathrm{mg}$ $\mathrm{ml}^{-1}$. The twofold dilution of the compounds and reference drug were prepared $(20,10,5.0,2.5$, $1.25,0.625,0.31,0.15,0.07,0.03,0.019,0.01,0.005>) \mathrm{mg} \mathrm{ml}^{-1}$. Antibacterial activities of the bacterial strains were carried out in Muller- Hinton broth (Difco) medium, at pH 6.9, with an inoculum of $(1-2) \times 10^{3}$ cells $\mathrm{ml}^{-1}$ by the spectrophotometric method and an aliquot of $100 \mu 1$ was added to each tube of the serial dilution. The chemical compounds-broth medium serial tube dilutions inoculated with each bacterium were incubated on a rotary shaker at $37{ }^{\circ} \mathrm{C}$ for $24 \mathrm{~h}$ at $150 \mathrm{rpm}$. The minimum inhibitory concentrations of the chemical compounds were recorded as the lowest concentration of each chemical compounds in the tubes with no growth (i.e. no turbidity) of inoculated bacteria.

Table 1. Antimicrobial Activity

\begin{tabular}{ccccc}
\hline & \multicolumn{4}{c}{ Minimum Inhibitory Concentration in mgml $^{-1}$} \\
\cline { 2 - 5 } Sr.No. & S. aureus & B. subtillis & E. coli & S. typhosa \\
\hline 10a & 0.03 & 0.015 & 0.15 & 1.25 \\
10b & 0.31 & 0.07 & 1.25 & 0.625 \\
10c & 0.625 & 0.015 & 5.0 & 2.5 \\
10d & 2.5 & 2.5 & 0.03 & 5.0 \\
10e & 0.15 & 5.0 & 2.5 & 5.0 \\
10f & 0.15 & 0.625 & 1.25 & 1.25 \\
10g & 2.5 & 2.5 & 5.0 & 0.625 \\
13h & - & 10 & 1.25 & - \\
13i & 2.5 & - & 0.625 & 5.0 \\
13j & 1.25 & - & 2.5 & 10 \\
13k & 1.25 & 5.0 & 2.5 & 1.25 \\
13l & 2.5 & 1.25 & 0.015 & 2.5 \\
13m & 0.015 & 0.625 & 2.5 & 0.625 \\
13n & 0.625 & 0.07 & 5.0 & 2.5 \\
13o & 2.5 & 2.5 & 10 & 1.25 \\
\hline
\end{tabular}


Table 1. Continued

\begin{tabular}{ccccc}
\hline \multirow{2}{*}{ Sr.No. } & \multicolumn{4}{c}{ Minimum Inhibitory Concentration in mgml $^{-1}$} \\
\cline { 2 - 5 } & S. aureus & B. subtillis & E. coli & S. typhosa \\
\hline $\mathbf{1 3 p}$ & 2.5 & 5.0 & 5.0 & 0.015 \\
$\mathbf{1 3 q}$ & 1.25 & 1.25 & 2.25 & 5.0 \\
$\begin{array}{c}\text { Ampicillin } \\
\text { trihydrate }\end{array}$ & 0.019 & 0.005 & 0.005 & 0.01 \\
\hline
\end{tabular}

\section{Conclusions}

A series of quinolinyl chalcones were prepared and tested for their in vitro antibacterial activity against the four strains of bacteria (gram +ve, gram -ve). Five compounds of the obtained series showed high in vitro antimicrobial activity. Compound (10a) showed excellent activity against Staphylococcus aureus and Bacillus subtillis, compound (10b) showed good activity against Bacillus subtillis, compound (10c) showed excellent activity against Bacillus subtillis, compound (13l) showed excellent activity against Escherichia coli and compound (13p) showed excellent activity against Salmonella typhosa. The presence of more than one electron-withdrawing group on the aromatic ring in Series 1 in general increased the antimicrobial activity compared to compounds with electron-donating groups. The presence of electron-donating group on the aromatic ring in Series 2 in general decreased the antimicrobial activity compared to compounds with electron-withdrawing groups. Based upon the results, it will also be necessary to optimize the by substituting a series of electron-withdrawing groups on the aromatic ring and selectively modifying the quinoline nucleus. The substitution in the C-2, C-3, and C-4 positions in Series 1 and $\mathrm{C}-2$ and $\mathrm{C}-4$ positions in Series 2 in of the phenyl ring seems to be very important for antibacterial effect, as well as the presence and the position of -NHCO-group in the connecting linker between the aromatic ring seems to be very important for antibacterial effect.

\section{Acknowledgements}

The authors are thankful to the Head of the Chemistry Department, Dr. K. R. Desai and Bioscience Department of Veer Narmad South Gujarat University, Surat. The authors also express their sincere thanks to the SAIF, Punjab University, Chandigarh for spectral analysis.

\section{References}

1. Ferlini, C.; Disterfand, M.; Valenti, P. Cancer Therapy Pharmacol. 2000, 46, 305.

2. Liu, M.; Wilairat, P.; Crajt, S. Biorg. Med. Chem. Lett. 2003, 11, 2729. 
3. Leon, C.; Gut, J.; Rosenthal, P. J. J. Med. Chem. 2005, 48, 3654.

4. Ram, V.; Saxena, A.; Srivastava, S. Bioorg. Med. Chem. Lett. 2000, 10, 2159.

5. Dominguez, J.; Charris, J. Eur. J. Med. Chem. 2001, 36,555.

6. Charris, J.; Dominguez, J. Eur. J. Med. Chem. 2005, 40, 875.

7. Dominguez, J.; Lobo, G. Eur. J. Med. Chem. 2001, 36, 555.

8. Dominguez, J.; Basante, W.; Charris, J.; Riggione, F. Fermaco 1996, 51, 407.

9. Patel, R.; Desai, K.; Chikhalia, K. J. Ind. Chem. Soc. 2003, 80, 138.

10. Anjaneyulla, A.; Sudharaki, G. J. Ind. Chem. 1995, 34(B), 933.

11. Desai, K.; Patel, R.; Chikhalia, K. J. Ind. Chem. 2006, 45(B), 773.

12. Ludovici, D.; Janseen, P. Biorg. and Med. Chem. Lett. 2004, 11, 2335.

13. Srivastava, A.; Singh, R. Ind. J. chem. 2005, 44B, 1868.

14. Patel R. B.; Ph.D. Thesis, VNSGU, 2003.

15. Clause, G. W Understanding Microbes: A Laboratory Textbook for Microbiology, W. H. Freeman and Company: New York, USA, 1989. 